fundamentals", says Austin.

NCI officials say that the latest awards, along with two rounds of funding planned for later this year or next year, show the institute's continuing commitment to the interdisciplinary approach. "The fact that this programme is renewed, while it's not in the same original form, is still an indication of support," says Larry Nagahara, a former director of the programme who left the NCI for Johns Hopkins University in Baltimore, Maryland, this month. Officials insist that there has been no move away from physics, although the programme also embraces related fields such as engineering and applied mathematics. "We're sort of agnostic on the spectrum of research that people are working on," says current programme head Sean Hanlon.

The PS-OC programme was largely the brainchild of Anna Barker, who in 2007-08, as a deputy director at the NCI, set up workshops that helped to lay the programme's intellectual foundation. She and other proponents pointed out that although billions of dollars of research investment into drugs and therapies have reduced mortality for some cancers, they have not produced a fundamental understanding of the disease. Programme leaders proposed to open a new front in the war on cancer by recruiting physicists to study cancer as a physical rather than strictly biological phenomenon.

\section{A DIFFERENT PERSPECTIVE}

In 2009, the NCI gave grants averaging $\$ 2.5$ million a year for 5 years to 12 centres, each co-directed by a physical scientist and a cancer biologist. Some researchers attempted to re-envision cancer from the bottom up. For example, physicist Paul Davies of Arizona State University in Tempe, who along with Austin was involved in the initial programme workshops (see Nature 474, 20-22; 2011), has proposed that a cell becomes cancerous when it reverts to a primitive evolutionary state. He is investigating whether ancient genes become activated during cancer development (P.C. W. Davies and C. H. Lineweaver Phys. Biol. 8, 015001; 2011). Austin has explored the evolution of drug resistance by using microfluidic devices to expose tumour cells to chemical gradients (A. Wu et al. Proc. Natl Acad. Sci. USA 110, 16103-16108; 2013), and has suggested that cancer might result from environmental stress rather than from genetic mutations.

Others have sought to develop or refine mathematical or biophysical tools for cancer research. At the Dana-Farber Cancer Institute in Boston, Massachusetts, for example, researchers have built computer simulations to predict which genetic and cellular changes are most likely to lead to certain cancers, and which treatment approaches are most likely to succeed. Other centres have used advanced

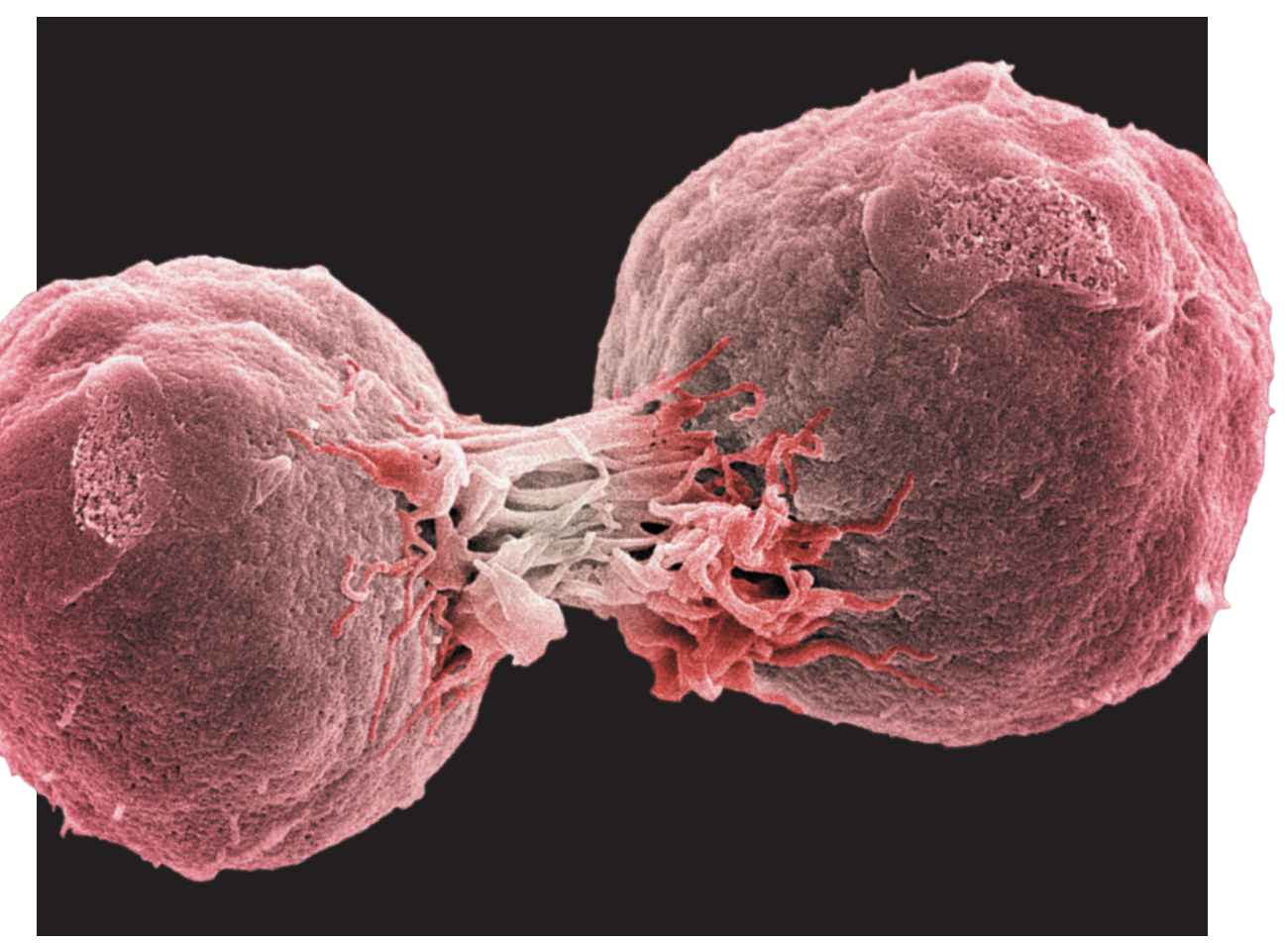

Cell division and other cancer processes are being studied by physicists looking for fundamental insights.

microscopy and spectroscopy. Such projects are valuable, but do not seek the kind of fundamental understanding of cancer that is the hallmark of the physics approach, says Herbert Levine, a physicist at Rice University in Houston, Texas, who studies cancer but has not received PS-OC funding.

The awards announced in June went to existing centres at Northwestern University in Chicago, Illinois, and Dana-Farber, as well as to two new ones - at Columbia University in New York City and the University of Pennsylvania in Philadelphia. Neither Austin nor Davies had their proposals funded. Those decisions may reflect the tangible results

"The lofty goal
of helping find
a new set of
directions in
biology - I don't
think they quite
got there."
produced by less paradigm-challenging projects, Levine says. He thinks that projects seeking fundamental breakthroughs, such as Austin's, need more time to achieve their visions. "The lofty goal of helping find a new set of directions in biology with the help of physicists, computer scientists, whatever - I don't think they quite got there."

Barker, who left the NCI in 2010 and is now at Arizona State, says that the PS-OCs have made progress in a number of areas, including understanding cancer evolution, predicting when a cell will become metastatic and developing biomarkers for cancer. But she agrees that five years was probably too short for the more ambitious efforts. "For these large consortia, it takes about the first three years to get them all working together, to get a common language in place, to get common core resources developed," she says. "In terms of judging the programme, I'd like to have seen it a couple years hence."

NCI programme managers say that the plan was always to reopen the funding competition after five years, rather than simply to extend existing sites. More researchers applied for the second round of funding, they say, and there was not enough money for everyone. But they point out that physical oncologists now have more funding options. "I think most people will find somewhere to have their work supported," says Hanlon, whether through future PS-OC awards, other NCI programmes or external sources.

Levine, for example, has funding from the state of Texas and has been involved in a partnership between the US National Science Foundation and private donors. The Francis Crick Institute, set to open this year in London, promises to bring more physicists into biomedical research (see Nature 509, 544-545; 2014). Austin and Davies say they may look overseas or to private foundations to continue their work.

NCI programme managers say that the diversification of funding sources shows that the field is gaining support and recognition. They also point to the journal Convergent Science Physical Oncology, launched in June by IOP Publishing of Bristol, UK, and to standing sessions on physics and the evolution of cancer at the American Physical Society's annual March meeting and at meetings of the American Association for Cancer Research. “Those types of sessions didn't exist five years ago - now you can find them at several of these meetings", says Nagahara. "That's a sign of success." - 